\title{
Asymptotics of the Spectral Density for Radial Dirac Operators with Divergent Potentials
}

\author{
By \\ Michael S. P. EAStham* and Karl Michael SCHMIDT**
}

\begin{abstract}
We study the asymptotics of the spectral density of one-dimensional Dirac systems on the half-line with an angular momentum term and a potential tending to infinity at infinity. The problem has two singular end-points; however, as the spectrum is simple, the derivative of the spectral matrix has only one non-zero eigenvalue which we take to be the spectral density. Our main result shows that, assuming sufficient regularity of the potential, there are no points of spectral concentration for large values of the spectral parameter outside a neighbourhood of a discrete set of exceptional points.
\end{abstract}

\section{$\S 1$. Introduction}

It is known that the one-dimensional Dirac operator

$$
-i \sigma_{2} \frac{d}{d x}+\sigma_{3}+q(x)
$$

where $\sigma_{2}=\left(\begin{array}{cc}0 & -i \\ i & 0\end{array}\right), \sigma_{3}=\left(\begin{array}{cc}1 & 0 \\ 0 & -1\end{array}\right)$, and $q$ is a locally integrable potential with the property that $\lim _{x \rightarrow \infty} q(x)=-\infty$, has a purely absolutely continuous spectrum covering the whole real line provided that $1 / q$ is of bounded variation ([4], [5]). In this situation, one may ask whether the absolutely continuous spectrum is essentially homogeneous or whether the spectral density has local

Communicated by T. Kawai. Received February 1, 2007.

2000 Mathematics Subject Classification(s): Primary 34L05; Secondary 34L40, 47E05, $81 \mathrm{Q} 10$.

* School of Computer Science, Cardiff University, Queen's Buildings, 5 The Parade, Cardiff CF24 3AA, UK.

** School of Mathematics, Cardiff University, Senghennydd Road, Cardiff CF24 4AG, UK. 
maxima, also called points of spectral concentration, which have been observed to be associated with resonances. A recent paper [3] addressed this question for the above operator on the half-line $[0, \infty)$ with a boundary condition at the regular end-point 0 . It was shown that for sufficiently regular potentials $q$ (including, in particular, thrice differentiable potentials of power or exponential growth) there are no points of spectral concentration above a certain value $\Lambda$ of the spectral parameter ([3, Theorem 4.1]). This result holds true irrespective of the boundary condition at 0 , but the actual shape of the spectral density function, and in particular whether it is eventually increasing or decreasing, does depend on the choice of boundary condition. Also, for two exceptional boundary conditions slightly stronger regularity of the potential is required ([3, Theorem 5.1]).

In the case of a one-dimensional Dirac operator with two singular endpoints, e.g. on the whole real line, the fundamental difficulty presents itself that the expansion formula for square-integrable functions in terms of eigensolutions makes use of a spectral matrix rather than just a spectral function. The latter suffices in the special case of a regular end-point because one of the solutions in a fundamental system can be discarded in view of the boundary condition. With a matrix-valued spectral density, however, it is not at all obvious how points of spectral concentration are to be defined. In practice, the one-dimensional Dirac operator arises from the three-dimensional Dirac operator with a spherically symmetric potential by separation of variables in spherical polar coordinates (cf. [7, Section 1.A]). It then takes the form

$$
-i \sigma_{2} \frac{d}{d r}+\sigma_{3}+\frac{k}{r} \sigma_{1}+q(r) \quad(r \in(0, \infty)),
$$

where $\sigma_{1}=\left(\begin{array}{ll}0 & 1 \\ 1 & 0\end{array}\right)$ and $k \in \mathbb{Z} \backslash\{0\}$. The additional angular momentum term $\frac{k}{r} \sigma_{1}$ introduces a singularity at the end-point 0 , which is in the limit-point case if the angular momentum term dominates the radial potential $q$ near 0 . The result that the Dirac operator has a purely absolutely continuous spectrum if $q$ tends to $-\infty$ and $1 / q$ is of bounded variation carries over to this case ([6]).

In the present paper, we study the asymptotic behaviour of the spectral density of this operator for large values of the spectral parameter. We find that, although there is a spectral matrix for the operator, one of the eigenvalues of its derivative vanishes identically - a reflection of the fact that the operator has a simple spectrum - and we show that the other eigenvalue, a non-negative function of the spectral parameter, can be considered as the spectral density. As always in the case of two singular end-points, the spectral matrix, and 
hence this spectral density, depends on the arbitrary choice of an intermediate point $c \in(0, \infty)$ at which the fundamental system used for the expansion formula is canonical. Our main result is that, for sufficiently regular potentials, the spectral density has no local maxima for values of the spectral parameter which are sufficiently large and outside a neighbourhood of a certain discrete set of exceptional points (Theorem 4 below). For large spectral parameter, these exceptional points are asymptotically evenly spaced with distance $\pi / c$. As the derivative of the spectral density changes sign between consecutive pairs of exceptional points, these are not just an artefact of our method but intrinsic to the problem; note, however, that their positions depend on the choice of the splitting point $c$.

The paper is organised as follows. In Section 2 we establish the existence of a particular solution $w$, square-integrable at 0 , of the Dirac equation

$$
\left(-i \sigma_{2} \frac{d}{d r}+m \sigma_{3}+\frac{k}{r} \sigma_{1}+q(r)\right) u(r)=\lambda u(r)
$$

and obtain the asymptotics of the solution, its Prüfer angle and their derivatives with respect to the spectral parameter as $r \rightarrow 0$ (Theorem 1). This provides an expression of the $\lambda$-derivative of the Prüfer angle in terms of the growth of the solution which is analogous to that for the regular case (Corollary 1 - cf. $[3,(2.8)])$. In order to find the asymptotics of this $\lambda$-derivative for large values of $\lambda$ (Theorem 2), we need a bound on the growth of the size of the solution $w$ which is uniform in $\lambda$ (Lemma 3), which we deduce by means of a scaling argument from the case $\lambda=1$ (Lemma 2). In Section 3, we use these results to derive a formula for the derivative of the spectral matrix. The fundamental idea is to average the spectral matrices for boundary value problems on $(0, b]$ over the boundary condition at $b$ and then pass to the limit $b \rightarrow \infty$ (Theorem $3)$. The structure of the spectral matrix then suggests our definition of the spectral function for this operator. In Section 4, we apply the method of repeated integrations by parts developed in [3] to the resulting integral formula for the spectral density. It turns out that by far fewer integrations by parts are necessary here than in the case of a regular end-point, due to the slower decay of the leading asymptotic term. On the other hand, the oscillations of the leading term lead to the appearance of exceptional values for the spectral parameter in our main result.

\section{§2. Existence and Asymptotic Properties of an $L^{2}(0, \cdot)$ Solution}

In this section, we show - as a preparatory step for the analysis in Sections 3 and 4 - that the Dirac system (2.3), with an angular momentum term which 
is dominant near 0 , has a distinguished solution $w(r, \lambda)$ small at 0 for each $\lambda \in \mathbb{R}$. While the existence of such a solution can be inferred fairly easily by applying the Levinson theorem, we are particularly interested in the properties of its $\lambda$-derivative and of the $\lambda$-derivative of its Prüfer angle in the asymptotic limit $\lambda \rightarrow \infty$. This last question requires a uniform estimate of the solutions of (2.3) with respect to $\lambda$.

We begin with a general observation about the Prüfer variables associated with a Dirac system, which in general takes the form

$$
\left(-i \sigma_{2} \frac{d}{d r}+m(r) \sigma_{3}+l(r) \sigma_{1}+q(r)\right) u(r)=\lambda u(r) .
$$

Setting

$$
u(r)=|u(r)|\left(\begin{array}{c}
\sin \vartheta(r) \\
\cos \vartheta(r)
\end{array}\right),
$$

we obtain the equivalent set of Prüfer equations

$$
\begin{aligned}
\vartheta^{\prime} & =\lambda-q+m \cos 2 \vartheta-l \sin 2 \vartheta, \\
(\log |u|)^{\prime} & =l \cos 2 \vartheta+m \sin 2 \vartheta .
\end{aligned}
$$

The structure of the right-hand side of the Prüfer equations gives rise to an intimate relationship between the size of the solution, measured by $|u|$, and the derivative of the Prüfer angle $\vartheta$, as shown in the following lemma.

Lemma 1. For $\lambda$ in a neighbourhood of $\lambda_{0} \in \mathbb{R}$, let $u(\cdot, \lambda)$ be a solution of the Dirac system on an interval $I, \vartheta(\cdot, \lambda)$ its Prüfer angle and $x_{0} \in I$. Assume that $\vartheta(x, \cdot)$ is differentiable at $\lambda_{0}$ for each $x \in I$. Then

$$
\frac{\partial \vartheta}{\partial \lambda}\left(x, \lambda_{0}\right)=\frac{\left|u\left(x_{0}, \lambda_{0}\right)\right|^{2}}{\left|u\left(x, \lambda_{0}\right)\right|^{2}} \frac{\partial \vartheta}{\partial \lambda}\left(x_{0}, \lambda_{0}\right)+\int_{x_{0}}^{x} \frac{\left|u\left(t, \lambda_{0}\right)\right|^{2}}{\left|u\left(x, \lambda_{0}\right)\right|^{2}} d t \quad(x \in I) .
$$

Proof. Differentiating the equation for $\vartheta$ with respect to $\lambda$, we find

$$
\left(\frac{\partial \vartheta}{\partial \lambda}\right)^{\prime}=-2(m \sin 2 \vartheta+l \cos 2 \vartheta) \frac{\partial \vartheta}{\partial \lambda}+1=1-2(\log |u|)^{\prime} \frac{\partial \vartheta}{\partial \lambda},
$$

where' denotes differentiation with respect to $x$. The assertion follows by solving this first-order linear differential equation.

In the remainder of Section 2, we shall assume the following general hypotheses.

$$
\text { Assume } m, q \in L_{\mathrm{loc}}^{1}[0, \infty) \text { and } l(r)=\frac{k}{r}(r>0) \text { with }|k| \geq \frac{1}{2} \text {. }
$$


Theorem 1. Suppose (H1) holds with $k \geq \frac{1}{2}$. Then for each $\lambda \in \mathbb{R}$, the Dirac equation

$$
\left(-i \sigma_{2} \frac{d}{d r}+m(r) \sigma_{3}+\frac{k}{r} \sigma_{1}+q(r)\right) u(r)=\lambda u(r)
$$

has a unique solution $w(\cdot, \lambda)$ with the property

$$
w(r, \lambda)=\left(\begin{array}{c}
o(1) \\
1+o(1)
\end{array}\right) r^{k} \quad(r \rightarrow 0) .
$$

Furthermore, $w(r, \cdot)$ is differentiable and

$$
\frac{\partial}{\partial \lambda} w(r, \lambda)=o\left(r^{k}\right) \quad(r \rightarrow 0) .
$$

The Prüfer angle $\vartheta$ of $w$ has the properties

$$
\lim _{r \rightarrow 0} \vartheta(r, \lambda)=0 \quad \text { and } \quad \lim _{r \rightarrow 0} \frac{\partial \vartheta}{\partial \lambda}(r, \lambda)=0 .
$$

Remarks. 1. By symmetry, an analogous statement holds for $k \leq-\frac{1}{2}$; then

$$
w(r, \lambda)=\left(\begin{array}{c}
1+o(1) \\
o(1)
\end{array}\right) r^{-k} \quad(r \rightarrow 0) .
$$

2. It is well-known that under the hypotheses of Theorem 1 the Dirac system is in the limit-point case at 0 , and indeed all solutions other than $w$ grow like $r^{-k}$ as $r$ approaches 0 . The asymptotics of the solution $w$ can be obtained directly from the Levinson Theorem ([2, Theorem 1.3.1]), but here we give a more detailed proof in order to estimate the $\lambda$-derivative as well.

Proof. Writing $w(r)=r^{k} z(r)$, we transform the Dirac system

$$
w^{\prime}(r)=\left(\begin{array}{cc}
-\frac{k}{r} & m-q+\lambda \\
m+q-\lambda & \frac{k}{r}
\end{array}\right) w(r)
$$

into

$$
z^{\prime}(r)=A(r) z(r)+R(r, \lambda) z(r)
$$

where

$$
A(r)=\left(\begin{array}{cc}
-\frac{2 k}{r} & 0 \\
0 & 0
\end{array}\right), \quad R(r, \lambda)=\left(\begin{array}{cc}
0 & m-q+\lambda \\
m+q-\lambda & 0
\end{array}\right) .
$$

The simplified equation

$$
\phi^{\prime}(r)=A(r) \phi(r)
$$


has the fundamental system

$$
\Phi(r)=\left(\begin{array}{cc}
r^{-2 k} & 0 \\
0 & 1
\end{array}\right)
$$

and by a formal application of the variation of constants formula

$$
z(r)=\Phi(r) \Phi^{-1}\left(r_{0}\right)+\Phi(r) \int_{r_{0}}^{r} \Phi^{-1} R z
$$

we obtain the integral equation for $z$ (choosing the value 1 for the second component of $z(0)$ )

$$
z(r)=\left(\begin{array}{l}
0 \\
1
\end{array}\right)+\int_{0}^{r}\left(\begin{array}{cc}
\left(\frac{s}{r}\right)^{2 k} & 0 \\
0 & 1
\end{array}\right) R(s, \lambda) z(s) d s .
$$

Note that because of (H1) $R$ is integrable over $[0, r]$, so $\int_{0}^{r}|R(s, \lambda)| d s \rightarrow 0$ $(r \rightarrow 0)$ locally uniformly in $\lambda$.

To solve (2.5) by successive approximation, define $z_{1}(r, \lambda):=\left(\begin{array}{l}0 \\ 1\end{array}\right)(r \geq$ $0, \lambda \in \mathbb{R})$ and recursively

$z_{j+1}(r, \lambda):=\left(\begin{array}{l}0 \\ 1\end{array}\right)+\int_{0}^{r}\left(\begin{array}{cc}\left(\frac{s}{r}\right)^{2 k} & 0 \\ 0 & 1\end{array}\right) R(s, \lambda) z_{j}(s, \lambda) d s \quad(r \geq 0, \lambda \in \mathbb{R}, j \in \mathbb{N})$.

Then, observing that $\left(\frac{s}{r}\right)^{2 k} \leq 1(s \in[0, r])$, we estimate

$$
\left|z_{2}(r, \lambda)-z_{1}(r, \lambda)\right| \leq \int_{0}^{r}|R(s, \lambda)| d s
$$

and

$$
\left|z_{j+1}(r, \lambda)-z_{j}(r, \lambda)\right| \leq \int_{0}^{r}|R(s, \lambda)|\left|z_{j}(r, \lambda)-z_{j-1}(r, \lambda)\right| d s \quad(j>1) .
$$

By induction, this yields

$$
\left|z_{j+1}(r, \lambda)-z_{j}(r, \lambda)\right| \leq \frac{1}{j !}\left(\int_{0}^{r}|R|\right)^{j} \quad(j \in \mathbb{N}) .
$$

As the series $\sum_{j=1}^{\infty} \frac{1}{j !}\left(\int_{0}^{r}|R|\right)^{j}$ is convergent to $e^{\int_{0}^{r}|R|}-1$, the sequence

$$
z_{n}(r, \lambda)=z_{1}(r, \lambda)+\sum_{j=1}^{n-1}\left(z_{j+1}(r, \lambda)-z_{j}(r, \lambda)\right)
$$


converges locally uniformly in $r \in[0, \infty)$ and $\lambda \in \mathbb{R}$ to a limit function $z(r, \lambda)$ satisfying

$$
\left|z(r, \lambda)-\left(\begin{array}{l}
0 \\
1
\end{array}\right)\right| \leq e^{\int_{0}^{r}|R(s, \lambda) d s|}-1 \rightarrow 0 \quad(r \rightarrow 0) .
$$

$z$ is a solution of the integral equation (2.5) and hence also of (2.4).

To estimate its derivative, we first observe that $z_{j}(r, \lambda)$ is differentiable with respect to $\lambda$. Indeed, this is trivial for $z_{1}$, and follows by induction for $z_{j+1}$, with

$$
\begin{aligned}
\frac{\partial}{\partial \lambda} z_{j+1}(r, \lambda)= & \int_{0}^{r}\left(\begin{array}{cc}
\left(\frac{s}{r}\right)^{2 k} & 0 \\
0 & 1
\end{array}\right) R(s, \lambda) \frac{\partial z_{j}}{\partial \lambda}(s, \lambda) d s \\
& +\int_{0}^{r}\left(\begin{array}{cc}
\left(\frac{s}{r}\right)^{2 k} & 0 \\
0 & 1
\end{array}\right)\left(\begin{array}{cc}
0 & 1 \\
-1 & 0
\end{array}\right) z_{j}(s, \lambda) d s .
\end{aligned}
$$

Setting $\mathcal{R}(s, \lambda):=\max \{1,|R(s, \lambda)|\}$, we find using $(2.6)$

$$
\begin{aligned}
& \left|\frac{\partial}{\partial \lambda} z_{j+1}(r, \lambda)-\frac{\partial}{\partial \lambda} z_{j}(r, \lambda)\right| \\
\leq & \int_{0}^{r}|R(s, \lambda)| \frac{\partial}{\partial \lambda} z_{j}(r, \lambda)-\frac{\partial}{\partial \lambda} z_{j-1}(r, \lambda)\left|d s+\int_{0}^{r}\right| z_{j}(s, \lambda)-z_{j-1}(s, \lambda) \mid d s \\
\leq & \int_{0}^{r} \mathcal{R}(s, \lambda)\left|\frac{\partial}{\partial \lambda} z_{j}(r, \lambda)-\frac{\partial}{\partial \lambda} z_{j-1}(r, \lambda)\right| d s \\
& +\int_{0}^{r} \mathcal{R}(s, \lambda) \frac{1}{(j-1) !}\left(\int_{0}^{s} \mathcal{R}\right)^{j-1} d s \\
\leq & \int_{0}^{r} \mathcal{R}(s, \lambda)\left|\frac{\partial}{\partial \lambda} z_{j}(r, \lambda)-\frac{\partial}{\partial \lambda} z_{j-1}(r, \lambda)\right| d s+\frac{1}{j !}\left(\int_{0}^{r} \mathcal{R}\right)^{j} .
\end{aligned}
$$

By induction, this implies the estimate

$$
\left|\frac{\partial}{\partial \lambda} z_{j+1}(r, \lambda)-\frac{\partial}{\partial \lambda} z_{j}(r, \lambda)\right| \leq \frac{1}{(j-1) !}\left(\int_{0}^{r} \mathcal{R}\right)^{j} \quad(j \in \mathbb{N}) .
$$

The series $\sum_{j=1}^{\infty} \frac{1}{(j-1) !}\left(\int_{0}^{r} \mathcal{R}\right)^{j}=\left(\int_{0}^{r} \mathcal{R}\right) e^{\int_{0}^{r} \mathcal{R}}$ is convergent, so the formally differentiated series for $z$,

$$
\frac{\partial z}{\partial \lambda}(r, \lambda)=\sum_{j=1}^{\infty}\left(\frac{\partial}{\partial \lambda} z_{j+1}(r, \lambda)-\frac{\partial}{\partial \lambda} z_{j}(r, \lambda)\right)
$$

is locally uniformly convergent. Hence $z$ is differentiable and

$$
\left|\frac{\partial z}{\partial \lambda}(r, \lambda)\right| \leq\left(\int_{0}^{r} \mathcal{R}\right) e^{\int_{0}^{r} \mathcal{R}} \rightarrow 0 \quad(r \rightarrow 0) .
$$


Finally, it is clear from the asymptotics of $w(r, \lambda)=r^{k} z(r, \lambda)$ that $\lim _{r \rightarrow 0} \vartheta(r, \lambda)$ $=0$. For the derivative, note that

$$
\frac{\partial}{\partial \lambda} \vartheta(r, \lambda)=\frac{w^{\top}\left(\begin{array}{cc}
0 & -1 \\
1 & 0
\end{array}\right) \frac{\partial}{\partial \lambda} w}{|w|^{2}}=\frac{z^{\top}\left(\begin{array}{cc}
0 & -1 \\
1 & 0
\end{array}\right) \frac{\partial}{\partial \lambda} z}{\left|z^{\top} z\right|}
$$

SO

$$
\left|\frac{\partial}{\partial \lambda} \vartheta(r, \lambda)\right| \leq \frac{|z|\left|\frac{\partial}{\partial \lambda} z\right|}{z^{\top} z} \rightarrow 0 \quad(r \rightarrow 0) .
$$

Theorem 1 allows us to pass to the limit $x_{0} \rightarrow 0$ in Lemma 1, leading to the following formula.

Corollary 1. Under the hypotheses of Theorem 1, the Prüfer angle $\vartheta$ of the solution $w$ satisfies

$$
\frac{\partial \vartheta}{\partial \lambda}(r, \lambda)=\frac{1}{|w(r, \lambda)|^{2}} \int_{0}^{r}|w(t, \lambda)|^{2} d t \quad(r>0, \lambda \in \mathbb{R}) .
$$

We would like to use Corollary 1 in order to derive the asymptotics of $\frac{\partial \vartheta}{\partial \lambda}(r, \lambda)$ for fixed $r$ as $\lambda \rightarrow \infty$. This requires a uniform bound on $\frac{|w(t, \lambda)|^{2}}{|w(r, \lambda)|^{2}}$ in $t \in(0, r]$ and $\lambda$. As the estimate in the proof of Theorem 1 is only exponential in $\lambda$, we need more precise asymptotics, where the dependence on the spectral parameter $\lambda$ is not treated as a perturbation, but included in the unperturbed problem. We begin with a simplified equation corresponding to $\lambda=1$.

Lemma 2. Let $\Psi$ be a fundamental system of the equation

$$
\psi^{\prime}(r)=\left(\begin{array}{rr}
-\frac{2 k}{r} & 1 \\
-1 & 0
\end{array}\right) \psi(r) .
$$

Then there exists a constant $C_{1}$ such that

$$
\left|\Psi(x) \Psi^{-1}(r)\right|<C_{1} \quad(0<r \leq x<\infty) .
$$

Proof. As the transfer matrix $\Psi(x) \Psi^{-1}(r)$ is independent of the choice of the fundamental system $\Psi$, we only need to prove the assertion for one particular fundamental system.

Setting $\chi(s)=\psi\left(\frac{1}{s}\right)$, we can apply Levinson's Theorem ([2, Theorem 1.3.1]) to the equivalent differential equation

$$
\chi^{\prime}(s)=\left(\begin{array}{cc}
\frac{2 k}{s} & -\frac{1}{s^{2}} \\
\frac{1}{s^{2}} & 0
\end{array}\right) \chi(s)
$$


to find that there are solutions $\chi_{1}, \chi_{2}$ of the asymptotic form

$$
\chi_{1}(s)=\left(\left(\begin{array}{l}
1 \\
0
\end{array}\right)+o(1)\right) s^{2 k}, \quad \chi_{2}(t)=\left(\left(\begin{array}{l}
0 \\
1
\end{array}\right)+o(1)\right) \quad(s \rightarrow \infty) .
$$

Thus (2.7) has a fundamental system $\Psi$ with asymptotics

$$
\Psi(r)=\left(\begin{array}{cc}
r^{-2 k}(1+o(1)) & o(1) \\
o\left(r^{-2 k}\right) & 1+o(1)
\end{array}\right) \quad(r \rightarrow 0) .
$$

The inverse satisfies

$$
\Psi^{-1}(r)=\left(\begin{array}{cc}
r^{2 k}(1+o(1)) & o\left(r^{2 k}\right) \\
o(1) & 1+o(1)
\end{array}\right) \quad(r \rightarrow 0),
$$

so

$$
\Psi(x) \Psi^{-1}(r)=\left(\begin{array}{cc}
\left(\frac{r}{x}\right)^{2 k}(1+o(1))+o(1) & \left(\frac{r}{x}\right)^{2 k} o(1)+o(1) \\
\left(\frac{r}{x}\right)^{2 k} o(1)+o(1) & \left(\frac{r}{x}\right)^{2 k} o(1)+1+o(1)
\end{array}\right) \quad(r, x \rightarrow 0) .
$$

Therefore there exists $\hat{r}>0$ such that $\Psi(x) \Psi^{-1}(r)$ is bounded uniformly with respect to $x \in(0, \hat{r}], r \in(0, x]$.

In order to treat larger values of $x$ and $r$, we apply the method of [3, Theorem 2.1], to the Dirac system

$$
w^{\prime}(r)=\left(\begin{array}{cc}
-\frac{k}{r} & 1 \\
-1 & \frac{k}{r}
\end{array}\right) w(r) \quad(r>0),
$$

which is equivalent to $(2.7)$ by $w(r)=r^{k} \psi(r)$. For $w=|w|\left(\begin{array}{c}\sin \theta \\ \cos \theta\end{array}\right)$ we have

$$
\theta^{\prime}(r)=1-\frac{k}{r} \sin 2 \theta(r), \quad(\log |w|)^{\prime}(r)=\frac{k}{r} \cos 2 \theta(r) .
$$

Observing that

$$
\left(\log \left(1-\frac{k}{r} \sin 2 \theta\right)\right)^{\prime}=\frac{k \sin 2 \theta}{r^{2}-k r \sin 2 \theta}-2(\log |w|)^{\prime},
$$

we find for $r_{2} \geq r_{1} \geq r_{0}:=2|k|$

$$
\begin{aligned}
\log \frac{\left|w\left(r_{2}\right)\right|^{2}}{\left|w\left(r_{1}\right)\right|^{2}}= & \int_{r_{1}}^{r_{2}} \frac{k \sin 2 \theta(r)}{r^{2}-k r \sin 2 \theta(r)} d r-\log \left(1-\frac{k}{r_{2}} \sin 2 \theta\left(r_{2}\right)\right) \\
& +\log \left(1-\frac{k}{r_{1}} \sin 2 \theta\left(r_{1}\right)\right),
\end{aligned}
$$

which is uniformly bounded (both above and below) with respect to $r_{1}, r_{2}$ and the choice of the solution $w$. 
Furthermore, $\log \frac{|w(r)|^{2}}{|w(\hat{r})|^{2}}$ is bounded uniformly for all solutions $w$ and $r \in$ $\left[\hat{r}, r_{0}\right]$, and we can infer the existence of a constant $C>1$ such that

$$
\frac{1}{C} \leq \frac{|w(r)|^{2}}{|w(\hat{r})|^{2}} \leq C
$$

for all $r \leq \hat{r}$.

In particular,

$$
\frac{|w(x)|^{2}}{|w(r)|^{2}}=\frac{|w(x)|^{2}}{|w(\hat{r})|^{2}} \frac{|w(\hat{r})|^{2}}{|w(r)|^{2}} \leq C^{2}
$$

for all $x \geq r \geq \hat{r}$. Consequently, for any $r \geq \hat{r}, x \geq r$ and any solution $\psi$ of

$$
\frac{|\psi(x)|^{2}}{|\psi(r)|^{2}}=\left(\frac{r}{x}\right)^{2 k} \frac{|w(x)|^{2}}{|w(t)|^{2}} \leq C^{2} .
$$

As $\psi(x)=\Psi(x) \Psi^{-1}(r) \psi(r)$, it follows that the transfer matrix $\Psi(x) \Psi^{-1}(r)$ is bounded uniformly in $x \geq \hat{r}, r \in[\hat{r}, x]$.

The case $r<\hat{r}<x$ can be treated by observing that

$$
\Psi(x) \Psi^{-1}(r)=\Psi(x) \Psi^{-1}(\hat{r}) \Psi(\hat{r}) \Psi^{-1}(r) .
$$

Lemma 3. Let $w(\cdot, \lambda)$ be the solution of Theorem 1. Then there exists $r_{0}>0$ such that $\frac{|w(t, \lambda)|^{2}}{|w(r, \lambda)|^{2}}$ is bounded uniformly in $r \in\left(0, r_{0}\right], t \in(0, r]$ and $\lambda>0$.

Proof. With $\Psi$ denoting the fundamental system of $(2.7), \Psi(r, \lambda):=$ $\Psi(\lambda r)(r>0, \lambda>0)$ will be a fundamental system of

$$
\psi^{\prime}(r)=\left(\begin{array}{rr}
-\frac{2 k}{r} & \lambda \\
-\lambda & 0
\end{array}\right) \psi(r)
$$

and $\left|\Psi(x, \lambda) \Psi^{-1}(t, \lambda)\right|=\left|\Psi(\lambda x) \Psi^{-1}(\lambda t)\right|<C_{1}$ for all $x>0, t \in(0, x]$ and $\lambda>0$, where $C_{1}$ is the constant of Lemma 2 .

Proceeding as in the first part of the proof of Theorem 1, but with

$$
A(r, \lambda)=\left(\begin{array}{cc}
-\frac{2 k}{r} & \lambda \\
-\lambda & 0
\end{array}\right), \quad R(r)=\left(\begin{array}{cc}
0 & m-q \\
m+q & 0
\end{array}\right)
$$

we obtain the integral equation for $z(r)=r^{-k} w(r)$,

$$
z(r, \lambda)=\left(\begin{array}{l}
0 \\
1
\end{array}\right)+\int_{0}^{r} \Psi(r, \lambda) \Psi^{-1}(t, \lambda) R(t) z(t) d t .
$$


Here $R$ is integrable over $[0, r]$ because of the hypotheses on $m$ and $q$, so solving the integral equation by successive approximation with $z_{1}(r, \lambda):=\left(\begin{array}{l}0 \\ 1\end{array}\right)$, we find in analogy to the proof of Theorem 1

$$
\left|z_{j+1}(r, \lambda)-z_{j}(r, \lambda)\right| \leq \frac{1}{j !}\left(C_{1} \int_{0}^{r}|R|\right)^{j} \quad(j \in \mathbb{N})
$$

and hence for the limit

$$
\left|z(r, \lambda)-\left(\begin{array}{l}
0 \\
1
\end{array}\right)\right| \leq e^{C_{1} \int_{0}^{r}|R|}-1
$$

The bound is independent of $\lambda$, and for sufficiently small $r_{0}$ we have $e^{C_{1} \int_{0}^{r}|R|}$ $1=: \mathcal{E}<1$.

Now let $r \in\left(0, r_{0}\right], t \in(0, r]$. Then

$$
|w(r, \lambda)|=r^{k}|z(r, \lambda)| \geq r^{k}(1-\mathcal{E})
$$

and similarly $|w(t, \lambda)| \leq t^{k}(1+\mathcal{E})$, so

$$
\frac{|w(t, \lambda)|^{2}}{|w(r, \lambda)|^{2}} \leq\left(\frac{t}{r}\right)^{2 k}\left(\frac{1+\mathcal{E}}{1-\mathcal{E}}\right)^{2} \leq\left(\frac{1+\mathcal{E}}{1-\mathcal{E}}\right)^{2}
$$

with bound independent of $t, r$ and $\lambda$.

After these preparations we are in a position to calculate the large- $\lambda$ asymptotics of the Prüfer angle of $w$, following [3, Theorem 2.2].

Theorem 2. Assume the hypotheses of Theorem 1 and $m, q \in$ $A C_{\mathrm{loc}}(0, \infty)$. Then

$$
\frac{\partial \vartheta}{\partial \lambda}(r, \lambda)=r(1+o(1)) \quad(\lambda \rightarrow \infty)
$$

for each $r \in(0, \infty)$.

Proof. Let $r \in(0, \infty)$. Using Corollary 1, we find

$$
\frac{1}{r}\left(r-\frac{\partial \vartheta}{\partial \lambda}(r, \lambda)\right)=\frac{1}{r} \int_{0}^{r}\left(1-\frac{|w(t, \lambda)|^{2}}{|w(r, \lambda)|^{2}}\right) d t
$$

Calculating the derivative of

$$
\log \frac{m \cos 2 \vartheta-\frac{k}{r} \sin 2 \vartheta-q+\lambda}{\lambda-q}
$$


and applying the Prüfer equations (2.1) and (2.2), we find

$$
\begin{aligned}
& \log \frac{|w(t, \lambda)|^{2}}{|w(r, \lambda)|^{2}}=\left.\log \frac{m \cos 2 \vartheta-\frac{k}{r} \sin 2 \vartheta-q+\lambda}{\lambda-q}\right|_{t} ^{r} \\
& -\int_{t}^{r}\left(\frac{m^{\prime} \cos 2 \vartheta+\frac{k}{s^{2}} \sin 2 \vartheta-q^{\prime}}{m \cos 2 \vartheta-\frac{k}{s} \sin 2 \vartheta-q+\lambda}+\frac{q^{\prime}(s)}{\lambda-q(s)}\right) d s \rightarrow 0 \quad(\lambda \rightarrow \infty)
\end{aligned}
$$

using monotone convergence in the integral: the absolute value of the numerators is integrable and the denominators are bounded and eventually increasing in $\lambda$.

The integrand in (2.8) is bounded uniformly in $\lambda$ (on $\left(0, r_{0}\right]$ by Lemma 3 , on $\left[r_{0}, r\right]$ by the above asymptotic estimate) and pointwise convergent to 0 for $t \in(0, r]$. Hence the assertion follows by Lebesgue's convergence theorem.

\section{§3. A Formula for the Spectral Function for Divergent Potentials}

As the Dirac system (2.3) is in the limit-point case at both end-points, the corresponding minimal operator is essentially self-adjoint and there is a unique self-adjoint realisation

$$
H=-i \sigma_{2} \frac{d}{d r}+m(r) \sigma_{3}+\frac{k}{r} \sigma_{1}+q(r) .
$$

In this section we obtain a formula for its spectral matrix in terms of the distinguished solution $w$ of Theorem 1. Recall that the generalised Fourier expansion formula associated with $H$ relies on the choice of a fundamental system of the eigenvalue equation (2.3). In the case of a regular end-point, one chooses one basis function to satisfy the boundary condition at that point, thus eliminating the second basis function from the expansion. In our present situation, however, both end-points 0 and $\infty$ are singular (and in the limitpoint case), and it is then customary to use the canonical fundamental system with respect to some arbitrarily chosen interior point $c \in(0, \infty)$, i.e. the pair of solutions $\left(v_{1}, v_{2}\right)$ with $v_{1}(c, \lambda)=\left(\begin{array}{l}1 \\ 0\end{array}\right), v_{2}(c, \lambda)=\left(\begin{array}{l}0 \\ 1\end{array}\right)$. Both basis functions enter the expansion formula, and correspondingly the measure of the Fourier integral takes the form of a $(2 \times 2)$ spectral matrix.

We assume, in addition to (H1), the following properties of $m$ and $q$, which in particular imply that the Dirac system has a purely absolutely continuous spectrum covering the whole real line (cf. [4], [6]).

$$
\begin{aligned}
& \text { Assume } m, q \in A C_{\mathrm{loc}}(0, \infty),\|m\|_{\infty} \leq \infty, \lim _{r \rightarrow \infty} q(r)=-\infty \\
& \qquad \int^{\infty} \frac{\left|q^{\prime}\right|}{q^{2}}<\infty \quad \text { and } \quad \int^{\infty} \frac{\left|m^{\prime}\right|}{|q|}<\infty
\end{aligned}
$$


Let us fix the splitting point $c \in(0, \infty)$ and $\lambda_{0}>\|m\|_{\infty}+\frac{|k|}{c}+\sup q$.

Theorem 3. The self-adjoint operator $H$ has a purely absolutely continuous spectrum in $\left(\lambda_{0}, \infty\right)$ with spectral density (calculated with respect to the splitting point c)

$$
\frac{d}{d \lambda} \varrho_{\infty}(\lambda)=\frac{w(c, \lambda) w(c, \lambda)^{\top}}{\pi|w(\infty, \lambda)|^{2}} \quad\left(\lambda \geq \lambda_{0}\right),
$$

where $w$ is the distinguished solution of Theorem 1.

Remarks. a) It will be shown in Lemma 4 that the limit $|w(\infty, \lambda)|=$ $\lim _{x \rightarrow \infty}|w(x, \lambda)|$ exists for all $\lambda \geq \lambda_{0}$.

b) The spectral density matrix has two eigenvalues, 0 and

$$
\frac{d}{d \lambda} \tilde{\varrho}(\lambda):=\frac{|w(c, \lambda)|^{2}}{\pi|w(\infty, \lambda)|^{2}}
$$

(The vanishing of one eigenvalue reflects the fact that $H$ has a simple spectrum.) The relationship between $\frac{d}{d \lambda} \varrho_{\infty}$ and $\frac{d}{d \lambda} \tilde{\varrho}$ is made explicit in

$$
\frac{d}{d \lambda} \varrho_{\infty}(\lambda)=\left(\begin{array}{cc}
\sin ^{2} \vartheta(c, \lambda) & \sin \vartheta(c, \lambda) \cos \vartheta(c, \lambda) \\
\sin \vartheta(c, \lambda) \cos \vartheta(c, \lambda) & \cos ^{2} \vartheta(c, \lambda)
\end{array}\right) \frac{d}{d \lambda} \tilde{\varrho}(\lambda)
$$

Thus, with $\hat{w}(x, \lambda):=\frac{w(x, \lambda)}{|w(c, \lambda)|}$, we have the expansion formula

$$
\begin{aligned}
f(x)= & \int_{\mathbb{R}} \sum_{\sigma, \tau=1}^{2}\left(\int_{0}^{\infty} f(s)^{\top} v_{\sigma}(s, \lambda) d s\right) v_{\tau}(x, \lambda)\left(d \varrho_{\infty}(\lambda)\right)_{\sigma \tau} \\
= & \int_{\mathbb{R}}\left(\int_{0}^{\infty} f(s)^{\top}\left(\sin \vartheta(c, \lambda) v_{1}(s, \lambda)+\cos \vartheta(c, \lambda) v_{2}(s, \lambda)\right)\right) \\
& \left(\sin \vartheta(c, \lambda) v_{1}(x, \lambda)+\cos \vartheta(c, \lambda) v_{2}(x, \lambda)\right) d \tilde{\varrho}(\lambda) \\
= & \int_{\mathbb{R}}\left(\int_{0}^{\infty} f(s)^{\top} \hat{w}(s, \lambda) d s\right) \hat{w}(x, \lambda) d \tilde{\varrho}(\lambda) \quad\left(f \in L^{2}(0, \infty)^{2}\right),
\end{aligned}
$$

in which $\tilde{\varrho}$ plays the role of a spectral function. Clearly $\tilde{\varrho}$ and the spectral matrix $\varrho_{\infty}$ depend on the choice of $c$.

c) It seems reasonable to identify points of spectral concentration with local maxima of $\frac{d}{d \lambda} \tilde{\varrho}(\lambda)$. Note that if $\frac{d}{d \lambda} \tilde{\varrho}(\lambda)$ is monotonic for large $\lambda$, the matrix entries of $\frac{d}{d \lambda} \varrho_{\infty}$ will not be monotonic, as $\vartheta(c, \cdot)$ is unbounded in $(3.2)$ by Theorem 2.

These observations motivate the following definition. 
Definition. We call $\tilde{\varrho}$ the spectral function of $H$ with respect to the splitting point $c$. Local maxima of $\frac{d}{d \lambda} \tilde{\varrho}$ will be called points of spectral concentration.

For the proof of Theorem 3, we need an asymptotic form of Theorem 2 (and the estimates involved) in the large- $x$ limit.

Lemma 4. Under the hypotheses (H2), the following statements hold true.

a) There exists a constant $C_{2}>1$ such that

$$
\frac{1}{C_{2}} \leq \frac{|w(r, \lambda)|}{|w(c, \lambda)|} \leq C_{2} \quad\left(r \geq c, \lambda \geq \lambda_{0}\right)
$$

b) $\frac{|w(r, \lambda)|}{|w(c, \lambda)|}=1+o(1) \quad(\lambda \rightarrow \infty) \quad$ with o-term uniform in $r \geq c$.

c) The limit $\frac{|w(\infty, \lambda)|}{|w(c, \lambda)|}=\lim _{r \rightarrow \infty} \frac{|w(r, \lambda)|}{|w(c, \lambda)|}$ exists and satisfies

$$
\frac{|w(\infty, \lambda)|}{|w(c, \lambda)|}=1+o(1) \quad(\lambda \rightarrow \infty)
$$

d) The o-term in the statement of Theorem 2 is uniform in $r \geq c$.

Proof. a) As in the proof of Theorem 2, we have for $r \geq c$

$$
\begin{aligned}
\log \frac{|w(r, \lambda)|^{2}}{|w(c, \lambda)|^{2}} & =\left.\log \frac{\lambda-q+m \cos 2 \vartheta-\frac{k}{r} \sin 2 \vartheta}{\lambda-q}\right|_{r} ^{c} \\
& +\int_{c}^{r} \frac{q^{\prime}\left(m \cos 2 \vartheta-\frac{k}{s} \sin 2 \vartheta\right)}{(\lambda-q)\left(\lambda-q+m \cos 2 \vartheta-\frac{k}{s} \sin 2 \vartheta\right)} d s \\
& +\int_{c}^{r} \frac{m^{\prime} \cos 2 \vartheta+\frac{k}{s^{2}} \sin 2 \vartheta}{\lambda-q+m \cos 2 \vartheta-\frac{k}{s} \sin 2 \vartheta} d s .
\end{aligned}
$$

All terms are bounded uniformly in $r \geq c$ and $\lambda \geq \lambda_{0}$.

b) For large $\lambda$, the logarithms in (3.3) are $\log \left(1+O\left(\lambda^{-1}\right)\right)=O\left(\lambda^{-1}\right)$; the integrands are bounded by the $\lambda$-independent integrable functions

$$
\frac{\left|q^{\prime}\right|\left(|m|+\frac{|k|}{c}\right)}{\left(\lambda_{0}-q-|m|-\frac{|k|}{c}\right)^{2}} \quad \text { and } \quad \frac{\left|m^{\prime}\right|+\frac{|k|}{c^{2}}}{\lambda_{0}-q-|m|-\frac{|k|}{c}},
$$

respectively, and pointwise convergent to 0 . The assertion follows by Lebesgue's convergence theorem. 
c) Pass to the limit $r \rightarrow \infty$ using the uniformity of the $\lambda$-asymptotics with respect to $r$.

d) By Lemma 1, Theorem 2 and repeated application of part b), we have

$$
\begin{aligned}
\frac{\partial \vartheta}{\partial \lambda}(r, \lambda) & =\frac{|w(c, \lambda)|^{2}}{|w(r, \lambda)|^{2}} \frac{\partial \vartheta}{\partial \lambda}(c, \lambda)+(r-c)+\int_{c}^{r}\left(\frac{|w(t, \lambda)|^{2}}{|w(c, \lambda)|^{2}} \frac{|w(c, \lambda)|^{2}}{|w(r, \lambda)|^{2}}-1\right) d t \\
& =c(1+o(1))+(r-c)(1+o(1))=r(1+o(1)) \quad(\lambda \rightarrow \infty),
\end{aligned}
$$

where the $o$-terms are uniform in $r$.

Proof of Theorem 3. By [1, Theorem 9.5.1], the spectral matrix with respect to the canonical fundamental system at $c$ is the limit of the spectral matrices with the same reference point $c$ for the regular problems on $(a, b)$, where $0<a<c<b<\infty$, as $a \rightarrow 0$ and $b \rightarrow \infty$. After the first limit process $(a \rightarrow 0)$, we have the spectral matrix for the boundary value problem on $(0, b)$ with boundary condition $u(b)^{\top}\left(\begin{array}{c}\cos \beta \\ -\sin \beta\end{array}\right)=0$ at $b$ with some $\beta$. The spectrum of this boundary value problem is purely discrete; in fact its eigenvalues are determined by the condition that $\vartheta(b, \lambda)=\beta \bmod \pi$.

Denoting by $u_{j}$ the orthonormal eigenfunctions, we have the expansion formula for $f \in L^{2}(0, \infty)^{2}$

$$
\begin{aligned}
f & =\sum_{j}\left(f, u_{j}\right) u_{j}=\sum_{j}\left(f, r_{j 1} v_{1}+r_{j 2} v_{2}\right) \\
& =\sum_{j}\left(r_{j 1}^{2} g_{1} v_{1}+r_{j 1} r_{j 2} g_{1} v_{2}+r_{j 2} r_{j 1} g_{2} v_{1}+r_{j 2}^{2} g_{2} v_{2}\right)=\int_{\mathbb{R}} \sum_{\sigma, \tau=1}^{2} g_{\sigma} v_{\tau} d \varrho_{\sigma \tau}(\lambda),
\end{aligned}
$$

where $g_{\sigma}(\lambda):=\left(f, v_{\sigma}(\cdot, \lambda)\right)(\sigma \in\{1,2\}), u_{j}=r_{j 1} v_{1}+r_{j 2} v_{2}(j \in \mathbb{N})$ and

$$
\varrho_{\sigma \tau}(\lambda):=\sum_{\lambda_{j} \text { e.v. in }\left(\lambda_{0}, \lambda\right]} r_{j \sigma} r_{j \tau}=\left(\sum_{\lambda_{j} \text { e.v. in }\left(\lambda_{0}, \lambda\right]} r_{j} r_{j}^{\top}\right)_{\sigma \tau}
$$

is the $(\sigma, \tau)$-component of the spectral matrix.

As $u_{j}$ is square-integrable, it must be a multiple of $w\left(\cdot, \lambda_{j}\right)$, or more specifically,

$$
u_{j}(x)=\frac{w\left(x, \lambda_{j}\right)}{\sqrt{\int_{0}^{b}\left|w\left(t, \lambda_{j}\right)\right|^{2} d t}} .
$$

Since $w(x, \lambda)=w_{1}(c, \lambda) v_{1}(x, \lambda)+w_{2}(c, \lambda) v_{2}(x, \lambda)$, this implies

$$
r_{j 1}=\frac{w_{1}\left(c, \lambda_{j}\right)}{\sqrt{\int_{0}^{b}\left|w\left(t, \lambda_{j}\right)\right|^{2} d t}}, r_{j 2}=\frac{w_{2}\left(c, \lambda_{j}\right)}{\sqrt{\int_{0}^{b}\left|w\left(t, \lambda_{j}\right)\right|^{2} d t}} .
$$


Therefore the spectral matrix for the boundary value problem on $(0, b)$ is

$$
\varrho_{b}(\lambda)=\sum_{\lambda_{j} \text { e.v. in }\left(\lambda_{0}, \lambda\right]} \frac{w\left(c, \lambda_{j}\right) w\left(c, \lambda_{j}\right)^{\top}}{\int_{0}^{b}\left|w\left(t, \lambda_{j}\right)\right|^{2} d t} \quad\left(\lambda \geq \lambda_{0}\right) .
$$

To study the limit $b \rightarrow \infty$, consider the spectral matrix averaged with respect to the boundary condition angle $\beta$ at $b$,

$$
\int_{0}^{\pi} \varrho_{b}(\Lambda) d \beta=\int_{0}^{\pi} \sum_{\lambda_{j} \text { e.v. in }\left(\lambda_{0}, \lambda\right]} \frac{w\left(c, \lambda_{j}\right) w\left(c, \lambda_{j}\right)^{\top}}{\int_{0}^{b}\left|w\left(t, \lambda_{j}\right)\right|^{2} d t} d \beta .
$$

As noted above, the eigenvalues are characterised by $\vartheta\left(b, \lambda_{j}\right)=\beta \bmod \pi$. By Corollary $1, \vartheta(b, \cdot)$ is strictly increasing. Hence the $j$ th eigenvalue branch $\Lambda_{j}(\beta)$ satisfies

$$
d \Lambda_{j}(\beta)=\frac{d \beta}{\frac{\partial \vartheta}{\partial \lambda}\left(b, \Lambda_{j}(\beta)\right)}
$$

substituting $\lambda=\Lambda_{k}(\beta)$ in the integral and using Corollary 1, we obtain

$$
\int_{0}^{\pi} \varrho_{b}(\Lambda) d \beta=\int_{\lambda_{0}}^{\Lambda} \frac{w\left(c, \lambda_{j}\right) w\left(c, \lambda_{j}\right)^{\top}}{\int_{0}^{b}\left|w\left(t, \lambda_{j}\right)\right|^{2} d t} \frac{\partial \vartheta}{\partial \lambda}(b, \lambda) d \lambda=\int_{\lambda_{0}}^{\Lambda} \frac{w\left(c, \lambda_{j}\right) w\left(c, \lambda_{j}\right)^{\top}}{\left|w\left(b, \lambda_{j}\right)\right|^{2}} d \lambda .
$$

Thus the averaged spectral matrix is locally absolutely continuous with density

$$
\frac{w\left(c, \lambda_{j}\right) w\left(c, \lambda_{j}\right)^{\top}}{\left|w\left(b, \lambda_{j}\right)\right|^{2}}
$$

The spectral matrix for the operator $H$ with respect to the splitting point $c$ will now be the limit

$$
\varrho_{\infty}(\lambda)=\lim _{b \rightarrow \infty} \varrho_{b}(\lambda)
$$

with any boundary condition $\beta$. Therefore we would like to pass to the limit $b \rightarrow \infty$ on both sides of (3.4).

For the right-hand side, we note that

$$
\frac{w\left(c, \lambda_{j}\right) w\left(c, \lambda_{j}\right)^{\top}}{\left|w\left(c, \lambda_{j}\right)\right|^{2}}
$$

is independent of $b$. Using Lemma 4 a) and c), we can apply Lebesgue's convergence theorem to calculate

$$
\begin{aligned}
\lim _{b \rightarrow \infty} \int_{\lambda_{0}}^{\Lambda} \frac{w\left(c, \lambda_{j}\right) w\left(c, \lambda_{j}\right)^{\top}}{\left|w\left(b, \lambda_{j}\right)\right|^{2}} d \lambda & =\lim _{b \rightarrow \infty} \int_{\lambda_{0}}^{\Lambda} \frac{w\left(c, \lambda_{j}\right) w\left(c, \lambda_{j}\right)^{\top}}{\left|w\left(c, \lambda_{j}\right)\right|^{2}}\left(\frac{|w(c, \lambda)|}{|w(b, \lambda)|}\right)^{2} d \lambda \\
& =\int_{\lambda_{0}}^{\Lambda} \frac{w\left(c, \lambda_{j}\right) w\left(c, \lambda_{j}\right)^{\top}}{\left|w\left(\infty, \lambda_{j}\right)\right|^{2}} d \lambda .
\end{aligned}
$$


On the left-hand side of (3.4) we estimate $\varrho_{b}(\Lambda)$ uniformly with respect to $b$ and $\beta$. Choosing for any $\beta \in[0, \pi)$ integers $K, L$ such that $\Lambda_{K}(\beta)$ is the smallest, $\Lambda_{L}(\beta)$ the largest eigenvalue in $\left(\lambda_{0}, \Lambda\right]$, we have by Lemma 1 and Lemma 4 a)

$$
\begin{aligned}
(L-K) \pi & =\vartheta\left(b, \Lambda_{L}(\beta)\right)-\vartheta\left(b, \Lambda_{K}(\beta)\right) \\
& =\int_{\Lambda_{K}(\beta)}^{\Lambda_{L}(\beta)} \frac{\partial \vartheta}{\partial \lambda}(b, \lambda) d \lambda \\
& =\int_{\Lambda_{K}(\beta)}^{\Lambda_{L}(\beta)}\left(\frac{|w(c, \lambda)|^{2}}{|w(b, \lambda)|^{2}} \frac{\partial \vartheta}{\partial \lambda}(c, \lambda)+\int_{c}^{b} \frac{|w(c, \lambda)|^{2}}{|w(b, \lambda)|^{2}} d t\right) d \lambda \\
& \leq C_{2}^{2}\left(\vartheta(c, \Lambda)-\vartheta\left(c, \lambda_{0}\right)\right)+C_{2}^{4}(b-c)\left(\Lambda-\lambda_{0}\right) .
\end{aligned}
$$

As the number of eigenvalues in $\left(\lambda_{0}, \Lambda\right]$ for the boundary value problem on $(0, b)$ with boundary condition $\beta$ is $L-K+1$,

$$
\begin{aligned}
\left|\varrho_{\sigma \tau}(\Lambda)\right| & =\left|\sum_{\lambda_{j} \text { e.v. in }\left(\lambda_{0}, \lambda\right]} \frac{w_{\sigma}\left(c, \lambda_{j}\right) w_{\tau}\left(c, \lambda_{j}\right)}{\int_{0}^{b}\left|w\left(t, \lambda_{j}\right)\right|^{2} d t}\right| \\
& \leq(L-K+1) \frac{C_{2}^{2}}{b} \leq \frac{C_{2}^{4}}{\pi b}\left(\vartheta(c, \Lambda)-\vartheta\left(c, \lambda_{0}\right)\right)+\frac{C_{2}^{6}}{\pi b}(b-c)\left(\Lambda-\lambda_{0}\right)+\frac{C_{2}^{2}}{b},
\end{aligned}
$$

which is uniformly bounded in $b \geq c$ and $\beta$.

Hence by Lebesgue's convergence theorem

$$
\lim _{b \rightarrow \infty} \int_{0}^{\pi} \varrho(\Lambda) d \beta=\int_{0}^{\pi} \varrho_{\infty}(\Lambda) d \beta=\pi \varrho_{\infty}(\Lambda)
$$

and the formula (3.1) follows.

The remaining statement in Theorem 3 is a consequence of the fact that for any vector $v \in \mathbb{R}^{2}$, the matrix $v v^{\top}$ has eigenvalues $|v|^{2}$ (with eigenvector $v$ ) and 0 (with eigenvector orthogonal to $v$ ).

\section{§4. Intervals of No Spectral Concentration}

As shown in Theorem 3, the spectral density of the operator $H$ corresponding to the splitting point $c$ can be represented as

$$
\tilde{\varrho}^{\prime}(\lambda)=\frac{|w(c, \lambda)|^{2}}{\pi|w(\infty, \lambda)|^{2}}=\frac{1}{\pi} \exp \left(-2 \int_{c}^{\infty}\left(\frac{k}{s} \cos 2 \vartheta(s, \lambda)+m \sin 2 \vartheta(s, \lambda)\right) d s\right),
$$

where we have used the second Prüfer equation (2.2) for the second identity. In intervals where $\tilde{\varrho}^{\prime \prime}$ has a fixed sign, either positive or negative, $\tilde{\varrho}^{\prime}$ will be 
monotonic, and hence these intervals will not contain any points of spectral concentration.

Formally, we have

$$
\begin{aligned}
\tilde{\varrho}^{\prime \prime}(\lambda)= & -\frac{2}{\pi}\left(\frac{d}{d \lambda} \int_{c}^{\infty}\left(\frac{k}{s} \cos 2 \vartheta(s, \lambda)+m \sin 2 \vartheta(s, \lambda)\right) d s\right) \\
& \times e^{-2 \int_{c}^{\infty}\left(\frac{k}{s} \cos 2 \vartheta(s, \lambda)+m \sin 2 \vartheta(s, \lambda)\right) d s},
\end{aligned}
$$

and in order to ascertain the sign of $\tilde{\varrho}^{\prime \prime}$ it is sufficient to study the derivative term in brackets. However, as observed in an analogous situation in [3], it is advisable to transform the integral into a more obviously convergent one before proceeding to calculate its derivative with respect to $\lambda$.

For this purpose, we employ the first Prüfer equation (2.1) to write

$$
1=\frac{\vartheta^{\prime}+\frac{k}{r} \sin 2 \vartheta-m \cos 2 \vartheta}{Q},
$$

where we have abbreviated $Q(r, \lambda):=\lambda-q(r)$ (which tends to $\infty$ as $r \rightarrow \infty$ ). Then

$$
\begin{aligned}
& \int_{c}^{\infty}\left(\frac{k}{r} \cos 2 \vartheta+m \sin 2 \vartheta\right) d r \\
= & \int_{c}^{\infty}\left(\frac{k}{r} \cos 2 \vartheta+m \sin 2 \vartheta\right) \frac{\vartheta^{\prime}+\frac{k}{r} \sin 2 \vartheta-m \cos 2 \vartheta}{Q} d r \\
= & \frac{m(c) \cos 2 \vartheta(c, \lambda)-\frac{k}{c} \sin 2 \vartheta(c, \lambda)}{2 Q(c, \lambda)} \\
+ & \int_{c}^{\infty}\left(\frac{\frac{k}{r^{2}} \sin 2 \vartheta+\left(\frac{k^{2}}{r^{2}}-m^{2}\right) \sin 4 \vartheta-\frac{2 k m}{r} \cos 4 \vartheta}{2 Q}-\frac{q^{\prime}\left(\frac{k}{r} \sin 2 \vartheta-m \cos 2 \vartheta\right)}{2 Q^{2}}\right) d r
\end{aligned}
$$

after integration by parts. Differentiating the boundary term with respect to $\lambda$, we obtain

$\frac{-m(c) \sin 2 \vartheta(c, \lambda)-\frac{k}{c} \cos 2 \vartheta(c, \lambda)}{Q(c, \lambda)} \frac{\partial \vartheta}{\partial \lambda}(c, \lambda)-\frac{m(c) \cos 2 \vartheta(c, \lambda)-\frac{k}{c} \sin 2 \vartheta(c, \lambda)}{2 Q^{2}(c, \lambda)}$

As $\frac{\partial \vartheta}{\partial \lambda}(c, \lambda) \sim c(\lambda \rightarrow \infty)$ by Theorem 2 and $Q(c)$ grows linearly in $\lambda$, the second term is asymptotically small compared to the first, so the leading term for large $\lambda$ will be

$$
-\frac{m(c) c \sin 2 \vartheta(c, \lambda)+k \cos 2 \vartheta(c, \lambda)}{Q(c, \lambda)}
$$


We want to show that the integral in (4.1) is a higher-order term, i.e. that it will be $o\left(\lambda^{-1}\right)(\lambda \rightarrow \infty)$ after differentiation with respect to $\lambda$. Following the procedure of [3], we subject the integral to further integrations by parts until the decay order in $\lambda$ becomes sufficiently apparent. To simplify the book-keeping, we use the following convention.

Definition. Denote by $\mathcal{F}$ the space of linear combinations of the functions $r^{-\nu} \cos 2 \kappa \vartheta, r^{-\nu} \sin 2 \kappa \vartheta$ with $\nu \in \mathbb{N}_{0}, \kappa \in \mathbb{N}$.

Let $n, s_{1}, s_{2} \in \mathbb{N}_{0}$ and $f \in \mathcal{F}$. Then the integral

$$
I(\lambda)=\int_{c}^{\infty} \frac{\left(q^{\prime}\right)^{s_{1}}\left(q^{\prime \prime}\right)^{s_{2}} f}{Q^{n}}
$$

is said to be of type $\left(n ; s_{1}, s_{2}\right)$.

In the remainder of this section we assume, in addition to (H1), one of the following hypotheses, each of which already implies (H2). The hypotheses cover potentials of power growth $(\mathrm{P})$ and exponential growth $(\mathrm{E})$.

Condition (P). Assume $m=$ const $\neq 0, q, q^{\prime} \in A C_{\mathrm{loc}}(0, \infty)$. For $r$ in some interval $(X, \infty)$, let

$$
-q(r) \geq C_{3} r^{a} \quad \text { and } \quad\left|q^{(k)}(r)\right| \leq C_{4} r^{a-k} \quad(k \in\{1,2\}),
$$

where $a, C_{3}$ and $C_{4}$ are positive constants.

Condition (E). Assume $m=$ const $\neq 0, q, q^{\prime} \in A C_{\mathrm{loc}}(0, \infty)$. For $r$ in some interval $(X, \infty)$, let

$$
-q(r) \geq C_{3} r^{a}
$$

for some positive constants $a$ and $C_{3}$. Moreover, for some $\delta \in(0,1)$ and any $\varepsilon>0$, let

$$
\frac{r q^{(k)}}{|q|^{1+\delta}} \in L(0, \infty) \quad(k \in\{1,2\}) \quad \text { and } \quad \frac{q^{\prime}(r)}{|q(r)|^{1+\varepsilon}}=O(1) \quad(r \rightarrow \infty) .
$$

Under these conditions, we have the following asymptotic estimates for the $\lambda$-derivatives of integrals of type $\left(n ; s_{1}, s_{2}\right)$.

Lemma 5. Let I be an integral of type $\left(n ; s_{1}, s_{2}\right)$.

a) Assume that Condition (P) holds, $n \geq 2$ and $n-s_{1}-s_{2}+\frac{\left(s_{1}+2 s_{2}-2\right)}{a}>1$. Then $\frac{d}{d \lambda} I(\lambda)=o\left(\lambda^{-1}\right)(\lambda \rightarrow \infty)$. 
b) Assume that Condition (E) holds, $s_{2} \in\{0,1\}$ and

$$
\begin{aligned}
& n>s_{1}+s_{2}+1+\delta \quad \text { if } \quad s_{1}+s_{2} \neq 0 \text {, } \\
& n>1+\frac{2}{a} \quad \text { if } \quad s_{1}+s_{2}=0 \text {. }
\end{aligned}
$$

Then $\frac{d}{d \lambda} I(\lambda)=o\left(\lambda^{-1}\right)(\lambda \rightarrow \infty)$.

We omit the proof of this statement, which is completely analogous to the proof of [3, Lemma 3.2]. The main difference lies in the reduced regularity of $q$ and the less restrictive conditions, which we can admit here because only $o\left(\lambda^{-1}\right)$ is required (as opposed to $o\left(\lambda^{-2}\right)$ in [3]).

By applying the above idea of integration by parts repeatedly to the subsequent integrals, we eventually obtain integrals to which Lemma 5 applies. Each step in this procedure takes the following general form.

Lemma 6. Let $I(\lambda)$ be an integral of type $\left(n ; s_{1}, 0\right)$. If

$$
\lim _{r \rightarrow \infty} \frac{\left(q^{\prime}(r)\right)^{s_{1}}}{Q(r, \lambda)^{n+1}}=0
$$

then $I(\lambda)$ is a linear combination of the following terms:

(i) integrals of types $\left(n+1 ; s_{1}, 0\right),\left(n+1 ; s_{1}-1,1\right)$ and $\left(n+2 ; s_{1}+1,0\right)$,

(ii) a boundary term $\tilde{f}(c, \vartheta(c, \lambda)) Q(c, \lambda)^{-(n+1)}$, where $\tilde{f} \in \mathcal{F}$,

(iii) integrals $\int_{c}^{\infty} \frac{\left(q^{\prime}(r)\right)^{s_{1}} p\left(\frac{1}{r}\right)}{Q(r, \lambda)^{n+1}} d r$ with polynomial $p$.

Proof. Consider the case $f=a_{1} r^{-\nu_{1}} \cos 2 \kappa \vartheta+a_{2} r^{-\nu_{2}} \sin 2 \kappa \vartheta$ without loss of generality. By the same method of integration by parts as above, we find

$$
\begin{aligned}
I(\lambda)= & \int_{c}^{\infty} \frac{\left(q^{\prime}\right)^{s_{1}} f}{Q^{n}} \frac{\vartheta^{\prime}+\frac{k}{r} \sin 2 \vartheta-m \cos 2 \vartheta}{Q} d r \\
= & -\frac{\left(q^{\prime}(c)\right)^{s_{1}} \tilde{f}(c, \vartheta(c, \lambda))}{Q(c)^{n+1}}-\int_{c}^{\infty} \frac{s_{1}\left(q^{\prime}\right)^{s_{1}-1} q^{\prime \prime} \tilde{f}}{Q^{n+1}} \\
& -\int_{c}^{\infty} \frac{(n+1)\left(q^{\prime}\right)^{s_{1}+1} \tilde{f}}{Q^{n+2}}-\int_{c}^{\infty} \frac{\left(q^{\prime}\right)^{s_{1}} f_{1}}{Q^{n+1}} \\
& +\int_{c}^{\infty} \frac{\left(q^{\prime}\right)^{s_{1}}}{Q^{n+1}}\left(\left(\frac{a_{1} k}{2 r^{\nu_{1}+1}}-\frac{a_{2} m}{2 r^{\nu_{2}}}\right) \sin 2(\kappa+1) \vartheta\right. \\
& -\left(\frac{a_{1} m}{2 r^{\nu_{1}}}-\frac{a_{2} k}{2 r^{\nu_{2}+1}}\right) \cos 2(\kappa+1) \vartheta
\end{aligned}
$$




$$
\begin{aligned}
& -\left(\frac{a_{1} k}{2 r^{\nu_{1}+1}}+\frac{a_{2} m}{2 r^{\nu_{2}}}\right) \sin 2(\kappa-1) \vartheta \\
& \left.-\left(\frac{a_{1} m}{2 r^{\nu_{1}}}-\frac{a_{2} k}{2 r^{\nu_{2}+1}}\right) \cos 2(\kappa-1) \vartheta\right) d r .
\end{aligned}
$$

Here we have used $f \vartheta^{\prime}=\tilde{f}+f_{1}$, with

$$
\begin{aligned}
\tilde{f} & =\frac{a_{1}}{2 \kappa r^{\nu_{1}}} \sin 2 \kappa \vartheta-\frac{a_{2}}{2 \kappa r^{\nu_{2}}} \cos 2 \kappa \vartheta, \\
f_{1} & =\frac{\nu_{1} a_{1}}{2 \kappa r^{\nu_{1}+1}} \sin 2 \kappa \vartheta-\frac{\nu_{2} a_{2}}{2 \kappa r^{\nu_{2}+1}} \cos 2 \kappa \vartheta .
\end{aligned}
$$

Now we can formulate our main result.

Theorem 4. Assume the general hypotheses (H1) and in addition either (P) or $(\mathrm{E})$. Then

$$
\text { (4.3) } \pi \varrho^{\prime \prime}(\lambda)=-\frac{k \cos 2 \vartheta(c, \lambda)+m c \sin 2 \vartheta(c, \lambda)}{\lambda}+o\left(\frac{1}{\lambda}\right) \quad(\lambda \rightarrow \infty) .
$$

In particular, if we denote by $\left(\lambda_{j}\right)_{j \in \mathbb{Z}}$ the increasing sequence of values of $\lambda$ such that

$$
\tan 2 \vartheta(c, \lambda)=-\frac{k}{m c}
$$

then for each $\varepsilon>0$ there is $\Lambda_{0}$ such that there are no points of spectral concentration in $\left(\Lambda_{0}, \infty\right) \backslash \bigcup_{j \in \mathbb{Z}}\left(\lambda_{j}-\varepsilon, \lambda_{j}+\varepsilon\right)$.

Remarks. a) In view of Theorem 2 , the $\lambda_{j}$ are asymptotically evenly spaced with distance $\lambda_{j+1}-\lambda_{j} \sim \frac{\pi}{c}$.

b) For large $|k|, \arctan \frac{k}{m c} \sim \pm \frac{\pi}{2}$, so the $\lambda_{j}$ converge as $k \rightarrow \pm \infty$. This may be of interest for the three-dimensional spherically symmetric Dirac operator.

Proof of Theorem 4. As seen in the beginning of this section, integration by parts turns the integral

$$
\int_{c}^{\infty}\left(\frac{k}{r} \cos 2 \vartheta(r, \lambda)+m \sin 2 \vartheta(r, \lambda)\right) d r
$$

into a sum of a boundary term and integrals of types $(1 ; 0,0)$ and $(2 ; 1,0)$. As we recursively apply Lemma 6 , the new integrals turn into integrals of types summarised in Table 1. 
Table 1. Types of integrals arising in the proof of Theorem 4.

\begin{tabular}{|l|l|}
\hline integral type & generates types \\
\hline$(0 ; 0,0)$ & $(1 ; 0,0),(2 ; 1,0)$ \\
\hline$(1 ; 0,0)$ & $(2 ; 0,0),(3 ; 1,0)^{E}$ \\
\hline$(2 ; 0,0)$ & $(3 ; 0,0),(4 ; 1,0)^{E}$ \\
$(2 ; 1,0)$ & $(3 ; 1,0)^{E},(3 ; 0,1)^{E, P},(4 ; 2,0)^{E, P}$ \\
\hline$(3 ; 0,0)$ & $(4 ; 0,0),(5 ; 1,0)^{E}$ \\
$(3 ; 1,0)$ & $(4 ; 1,0)^{E},(4 ; 0,1)^{E, P},(5 ; 2,0)^{E, P}$ \\
\hline
\end{tabular}

The superscript $P$ or $E$ indicates that integrals of this type satisfy the hypotheses of Lemma 5 under condition $(\mathrm{P})$ or $(\mathrm{E})$, respectively; then there is no further need to apply Lemma 6 to them. Clearly the process stops very quickly except for integrals of types $(n ; 0,0)$ (for which we need to continue until $n>1+\frac{2}{a}$ ) and, under condition $(\mathrm{P})$ only, $(n ; 1,0)$ (where we continue until $\left.n>2+\frac{1}{a}\right)$.

The boundary terms, apart from the very first one already dealt with above, are of the type

$$
\frac{\tilde{f}(c, \vartheta(c, \lambda))}{Q^{n+1}(c, \lambda)}
$$

with $\tilde{f} \in \mathcal{F}$ and $n \in \mathbb{N}$. Their $\lambda$-derivative will therefore be of the type

$\frac{-(n+1) \tilde{f}(c, \vartheta(c, \lambda))}{Q^{n+2}(c, \lambda)}+\frac{\tilde{\tilde{f}}(c, \vartheta(c, \lambda)) 2 \frac{\partial \vartheta}{\partial \lambda}(c, \lambda)}{Q^{n+2}(c, \lambda)}=O\left(\lambda^{-(n+2)}\right)=o\left(\lambda^{-1}\right) \quad(\lambda \rightarrow \infty)$.

There is no integral of type (iii) after the first integration by parts. The next step produces

$$
\int_{c}^{\infty} \frac{k^{2}}{4 r^{3} Q^{2}} d r+\int_{c}^{\infty} \frac{\frac{k^{2}}{r^{2}}+m^{2}}{4 Q^{3}} q^{\prime} d r
$$

Now

$$
\left|\frac{d}{d \lambda} \int_{c}^{\infty} \frac{k^{2}}{4 r^{3} Q^{2}} d r\right| \leq \frac{1}{\left|\lambda-\sup _{[c, \infty)} q\right|^{3}} \int_{c}^{\infty} \frac{k^{2}}{8 r^{3}} d r=O\left(\lambda^{-3}\right)
$$

and

$$
\left|\frac{d}{d \lambda} \int_{c}^{\infty} \frac{\frac{k^{2}}{r^{2}}+m^{2}}{4 Q^{3}} q^{\prime} d r\right| \leq \frac{1}{\left|\lambda-\sup _{[c, \infty)} q\right|^{2}} \int_{c}^{\infty} \frac{\frac{k^{2}}{c^{2}}+m^{2}}{12}\left|\frac{q^{\prime}}{q^{2}}\right| d r=O\left(\lambda^{-2}\right) .
$$


Similarly, all subsequent terms of this nature are of higher order in $\frac{1}{\lambda}$.

Finally, we check that (4.2) is satisfied in all relevant cases, i.e. that

$$
\lim _{r \rightarrow \infty} \frac{q^{\prime}(r)^{s_{1}}}{Q(r, \lambda)^{n+1}}=0
$$

for $n \geq 1, s_{1} \in\{0,1\}$. For $s_{1}=0$ this is obvious; for $s_{1}=1$ we have

$$
\left|\frac{q^{\prime}(r)}{Q(r, \lambda)^{n+1}}\right| \leq \frac{C_{4} r^{a_{1}}}{\left(\lambda+c r^{a}\right)^{n+1}} \rightarrow 0 \quad(r \rightarrow \infty)
$$

in the case of Condition $(\mathrm{P})$ and

$$
\left|\frac{q^{\prime}(r)}{Q(r, \lambda)^{n+1}}\right|=\left|\frac{q^{\prime}(r)}{|q|^{1+\varepsilon}}\right| \frac{|q|^{1+\varepsilon}}{|\lambda-q|^{n+1}} \rightarrow 0 \quad(r \rightarrow \infty)
$$

in the case of Condition (E). Thus we have established (4.3).

Now let $\varepsilon>0$. By Theorem 2, there are $\Lambda_{1} \geq \lambda_{0}$ and $\alpha>0$ such that

$$
|k \cos 2 \vartheta(c, \lambda)+m c \sin 2 \vartheta(c, \lambda)| \geq \alpha \quad\left(\lambda>\Lambda_{1},\left|\lambda-\lambda_{j}\right| \geq \varepsilon(j \in \mathbb{Z})\right) .
$$

As we have just seen, there will then be $\Lambda_{0} \geq \Lambda_{1}$ such that $\left|\tilde{\varrho}^{\prime \prime}(\lambda)\right|>\frac{\alpha}{2 \pi \lambda}$ for all $\lambda>\Lambda_{0}$ such that $\left|\lambda-\lambda_{j}\right| \geq \varepsilon(j \in \mathbb{Z})$. As a consequence, $\tilde{\varrho}^{\prime}$ is monotonic (either increasing or decreasing) in each interval $\left(\lambda_{j}+\varepsilon, \lambda_{j+1}-\varepsilon\right.$ ) above $\Lambda_{0}$, so these intervals do not contain any points of spectral concentration.

\section{References}

[1] E. A. Coddington and N. Levinson, Theory of ordinary differential equations, McGrawHill Book Company, Inc., New York, 1955.

[2] M. S. P. Eastham, The asymptotic solution of linear differential systems, Oxford Univ. Press, New York, 1989.

[3] M. S. P. Eastham and K. M. Schmidt, Absence of high-energy spectral concentration for Dirac systems with divergent potentials, Proc. Roy. Soc. Edinburgh Sect. A 135 (2005), no. 4, 689-702.

[4] A. Erdélyi, Note on a paper by Titchmarsh, Quart. J. Math. Oxford Ser. (2) 14 (1963), $147-152$.

[5] K. M. Schmidt, Absolutely continuous spectrum of Dirac systems with potentials infinite at infinity, Math. Proc. Cambridge Philos. Soc. 122 (1997), no. 2, 377-384.

[6] K. M. Schmidt and O. Yamada, Spherically symmetric Dirac operators with variable mass and potentials infinite at infinity, Publ. Res. Inst. Math. Sci. 34 (1998), no. 3, $211-227$.

[7] J. Weidmann, Spectral theory of ordinary differential operators, Lecture Notes in Math., 1258, Springer, Berlin, 1987. 